\title{
Intraoperative Chemotherapy with a Novel Regimen Improved the Therapeutic Outcomes of Colorectal Cancer
}

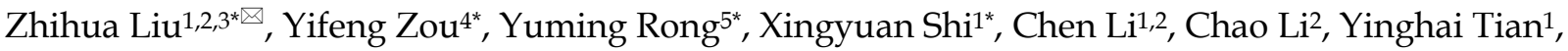

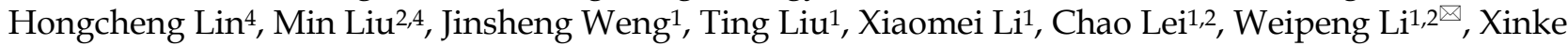 \\ Zhou $^{1 凶}$ \\ 1. Department of Center Laboratory, the Fifth Affiliated Hospital of Guangzhou Medical University, Guangzhou, Guangdong, 510799, China; \\ 2. Department of Anorectal Surgery, the Fifth Affiliated Hospital of Guangzhou Medical University, Guangzhou, Guangdong, 510799, China; \\ 3. Department of Surgery, the Sixth Affiliated Hospital of Shanghai JiaoTong University, Shanghai, 200233, China; \\ 4. Department of Colorectal Surgery, the Sixth Affiliated Hospital, Sun Yat-sen University, Guangzhou, Guangdong, 510655, China; \\ 5. Department of VIP, Sun Yat-Sen University Cancer Center, Guangzhou, Guangdong, 510060, China. \\ *Zhihua Liu, Yifeng Zou, Yuming Rong, and Xingyuan Shi are co-first authors.
}

$\triangle$ Corresponding authors: Dr. Zhihua Liu, MD., Ph.D., Department of Anorectal Surgery, the Fifth Affiliated Hospital of Guangzhou Medical University, 621 Gangwan Road, Guangzhou, Guangdong, 510799, China. Tel: +86 20 82281638; Fax: +86 20 82297393; Email: liuzhihua@gzhmu.edu.cn and Dr. Xinke Zhou, Department of Center Laboratory, the Fifth Affiliated Hospital of Guangzhou Medical University, Guangzhou, Guangdong, 510799, China. Email: xkzhou12@outlook.com and Mr. Weipeng Li, Department of Center Laboratory, the Fifth Affiliated Hospital of Guangzhou Medical University, 621 Gangwan Road, Guangzhou, Guangdong, 510799, China. Tel: +86 20 82281638; Fax: +86 20 82297393; Email: 1274328274@qq.com

(c) The author(s). This is an open access article distributed under the terms of the Creative Commons Attribution License (https://creativecommons.org/licenses/by/4.0/). See http:/ /ivyspring.com/terms for full terms and conditions.

Received: 2019.04.03; Accepted: 2019.08.20; Published: 2019.10.15

\begin{abstract}
Background: This study sought to evaluate the efficacy of a novel intraoperative chemotherapy (IOC) regimen that consists of hydroxycamptothecin, tumor necrosis factor (TNF), 5-fluorouracil (5-FU), and calcium folinate (CF) on the outcomes of colorectal cancer (CRC).

Methods: In total, 551 CRC patients who had undergone surgical resection were evaluated. Among these patients, 247 were treated with postoperative adjuvant chemotherapy, and 193 were treated with intraoperative chemotherapy. Of the CRC patients who underwent chemotherapy, 52 were treated with both postoperative adjuvant chemotherapy and intraoperative chemotherapy. Patients' characteristics, including age, sex, stage, differentiation, lymph node metastasis, surgical-pathological staging, tumor location, tumor size, and relapse-free survival, were collected.

Results: IOC for CRC therapy was associated with a more favorable survival prognosis $(\mathrm{HR}, 0.30,95 \% \mathrm{Cl}$, $0.19-0.48, P<0.001$ ) independent of other clinical covariates. CRC patients treated with IOC survived longer than patients who were not treated with IOC did during surgery $(\mathrm{P}<0.0001$, Kaplan-Meier log rank). Meanwhile, a Kaplan-Meier analysis demonstrated that individuals who received both IOC and POC survived longer than patients who received only POC: for stage II and stage III patients ( $P=0.0001$, Kaplan-Meier log rank), stage II patients alone $(P=0.02$, Kaplan-Meier log rank), and stage III patients alone $(P=0.046$, Kaplan-Meier log rank).

Conclusions: The therapeutic effects of colorectal cancer by intraoperative chemotherapy with a novel regimen were enhanced, which improved the prognosis of patients with CRC.
\end{abstract}

Key words: colorectal cancer, intraoperative chemotherapy, prognosis, therapy

\section{Introduction}

Colorectal cancer (CRC) is the third most common cancer and the second most common cause of cancer-related mortality in Western countries, where there are approximately 600,000 new cases annually[1]. Furthermore, recent decades have witnessed a rapid increase in CRC morbidity in rapidly developing countries, such as China[2]. Currently, surgery is the mainstay of curative 
treatment; however, cancer recurrence and metastasis following surgery are common[3]. Therefore, there is an urgent need to further improve CRC treatment.

In an attempt to prevent cancer recurrence and metastasis following surgery, postoperative adjuvant intra-arterial chemotherapeutic agents have been used[4-7]. However, postoperative adjuvant intraarterial chemotherapy does not significantly prolonged survival in all cases[8-9]. Intra-arterial chemotherapy is also associated with considerable complications and high costs[10-11]. Rashidi et al.[12] have suggested a new strategy of intraoperative chemotherapy (IOC) for treating resectable highly malignant human colon cancer liver metastasis using in vivo experiments. The results of these studies have demonstrated that IOC is an effective and convenient treatment strategy to prolong the survival of rats.

In the present study, we evaluated the efficacy of IOC on the therapeutic outcomes of CRC using a novel regimen.

\section{Methods}

\section{Patient selection}

Between 2001 and 2008, 551 CRC patients who had undergone surgical resection were assessed for eligibility. Cases of familial adenomatous polyposis or human nonpolyposis CRC were excluded from this study. Patients' characteristics, including age, sex, disease stage, differentiation, lymph node metastasis, surgical-pathological staging, tumor location, tumor size, and relapse-free survival were collected. The final follow-up date for all of the cases was September 1, 2016.

\section{Criteria for inclusion}

The following inclusion criteria were used for patients in this study: 1) age between 20 and $95 \mathrm{y}$; 2) a diagnosis confirmed by biopsy and histologic testing.

\section{Criteria for exclusion}

The exclusion criteria were as follows: 1) pregnancy, 2) clinically significant immunodeficiency, 3) evidence of infection.

\section{Intraoperative chemotherapy}

Intraoperative chemotherapy regimens consisting of hydroxycamptothecin, tumor necrosis factor (TNF), 5-fluorouracil (5-FU), and calcium folinate (CF) were administered during the operations. Hydroxycamptothecin was used for peritoneal irrigation during the operations, while TNF, 5-FU, and CF were given as intravenous injections.

\section{Postoperative adjuvant chemotherapy}

The analyses of the response to postoperative adjuvant chemotherapy included only TNM stage II and III patients because TNM stage I patients have excellent survival prognoses regardless of therapy, and treatment in stage IV is palliative. The chemotherapy regimens were primarily fluorouracil-based, with or without leucovorin, levamisole, or cisplatin.

\section{Statistical Analysis}

The statistical analyses were performed with GraphPad Prism software (GraphPad Prism Software, Version 5.01, GraphPad, San Diego, CA) and SPSS for Windows version 15.0.0 (SPSS, Inc., USA). Survival curves were generated using the Kaplan-Meier method, and the statistical analyses were performed using the log-rank test. Multivariate analyses were evaluated with Cox proportional hazards models. Statistical significance was defined as $\mathrm{P}<0.05$.

\section{Results}

\section{Assembly of Tumor Samples}

All of the 551 patients $(100 \%)$ included in the study were Chinese. Patients with familial adenomatous polyposis or human nonpolyposis CRC were excluded from this study. In total, 247 (44.83\%) patients were treated with postoperative adjuvant chemotherapy, and $193(35.03 \%)$ patients were treated with intraoperative chemotherapy. Of the CRC patients who received chemotherapy, 52 were treated with both postoperative adjuvant chemotherapy and intraoperative chemotherapy. In the study, 303 $(54.99 \%)$ patients were male and $248(45.01 \%)$ were female. Detailed clinical characteristics are listed in Table 1.

\section{The association of IOC and POC with Prognosis for CRC patients}

To determine whether the prognostic value of IOC was independent of other risk factors associated with clinical outcomes of CRC, a multivariate analysis was performed using the Cox proportional hazard model. The risk variables examined included IOC, POC, age, differentiation, lymph node metastasis, surgical-pathological staging, tumor location, and tumor size. These factors were generally known to significantly affect the outcome of CRC. In the univariate analysis, IOC (HR 0.33, 95\% CI, 0.23-0.48, $\mathrm{P}<0.001$ ), POC (HR 0.62, 95\% CI, 0.45-0.87, $\mathrm{P}=0.006$ ), TNM staging (HR 2.52, 95\% CI, 1.89-3.36, $\mathrm{P}<0.001$ ), lymph node metastasis (HR 1.70, 95\%CI, 1.28-2.25, $\mathrm{P}<0.001$ ), and differentiation ( $\mathrm{HR}$ 1.92, 95\% CI, 1.09-3.36, $\mathrm{P}=0.024)$ were significantly associated with survival, while age, gender, tumor location, and tumor size were not (Table 2). In the final multivariate Cox regression model, which included IOC, POC, TNM staging, lymph node metastasis, distant 
metastasis, tumor size, age, gender, differentiation, and tumor location, IOC for CRC therapy was associated with an improved survival prognosis (HR $0.30,95 \% \mathrm{CI}, 0.19-0.48, \mathrm{P}<0.001)$ independent of other clinical covariates (Table 2).

Table 1. Clinical characteristics of colorectal cancer patients.

\begin{tabular}{|c|c|}
\hline Clinical features & Number $(\%)^{a}$ \\
\hline \multicolumn{2}{|l|}{ Gender - no. ( $\%)$} \\
\hline Female & $248(45.01)$ \\
\hline Male & $303(54.99)$ \\
\hline \multicolumn{2}{|l|}{ Age - years } \\
\hline Median & 68 \\
\hline Range & 24-91 \\
\hline \multicolumn{2}{|l|}{ Location - no. $(\%)^{b}$} \\
\hline Ascending colon & $151(27.40)$ \\
\hline Transverse colon & $24(4.36)$ \\
\hline Descending colon & $48(8.71)$ \\
\hline Sigmoid colon & $141(25.59)$ \\
\hline Rectum & $182(33.03)$ \\
\hline Missing data & $10(1.81)$ \\
\hline \multicolumn{2}{|l|}{ TNM stage - no. $(\%)^{c}$} \\
\hline I & $53(9.62)$ \\
\hline II & $267(48.46)$ \\
\hline III & $192(34.85)$ \\
\hline IV & $22(3.99)$ \\
\hline Missing & $17(3.09)$ \\
\hline \multicolumn{2}{|l|}{ Pathological type - no. (\%) } \\
\hline Tubular adenocarcinoma & $442(80.22)$ \\
\hline Mucinous adenocarcinoma & $57(10.34)$ \\
\hline Mixed adenocarcinoma & $52(9.44)$ \\
\hline \multicolumn{2}{|l|}{ Tumor size - no. $(\%)$} \\
\hline$<5 \mathrm{~cm}$ & $245(44.46)$ \\
\hline$\geq 5 \mathrm{~cm}$ & $250(45.37)$ \\
\hline Missing data & $56(10.16)$ \\
\hline \multicolumn{2}{|c|}{ Lymph node metastasis - no. $(\%)$} \\
\hline Yes & $201(36.48)$ \\
\hline No & $337(61.16)$ \\
\hline Missing data & $13(2.36)$ \\
\hline \multicolumn{2}{|l|}{ Differentiation -no. (\%) } \\
\hline Well & $59(10.71)$ \\
\hline Moderate & $356(64.61)$ \\
\hline Poor & $136(24.68)$ \\
\hline \multicolumn{2}{|c|}{ Postoperative adjuvant chemotherapy - no. $(\%)^{\mathrm{d}}$} \\
\hline Yes & $247(44.83)$ \\
\hline No & $281(51.00)$ \\
\hline Missing data & $23(4.17)$ \\
\hline \multicolumn{2}{|c|}{ Intraoperative chemotherapy - no. $(\%)^{\mathrm{e}}$} \\
\hline Yes & $193(35.03)$ \\
\hline No & $331(60.07)$ \\
\hline Missing data & $27(4.90)$ \\
\hline \multicolumn{2}{|l|}{ Survival - no. $(\%)$} \\
\hline Median & 63.97 \\
\hline Range & $0.13-117.93$ \\
\hline \multicolumn{2}{|c|}{$\begin{array}{l}\text { aPercentages may not sum to } 100 \text { because of rounding. bTumor location was } \\
\text { available for } 542 \text { patients in the current study. Four patients had both rectal and } \\
\text { sigmoid colon cancer, and } 1 \text { patient had both descending colon and sigmoid colon } \\
\text { cancer. cTNM denotes tumor-node-metastasis. dDetailed information pertaining to } \\
\text { the receipt of postoperative adjuvant chemotherapy was available for } 247 \text { patients } \\
\text { in the current study. Chemotherapy was primarily fluorouracil based with or } \\
\text { without leucovorin, levamisole, or cisplatin. } \text { 'Detailed information pertaining to the } \\
\text { receipt of intraoperative chemotherapy was available for } 193 \text { patients in the current } \\
\text { study. The intraoperative chemotherapy regimens consisting of } \\
\text { hydroxycamptothecin, calcium folinate (TNF), 5-fluorouracil (5-FU), and calcium } \\
\text { folinate (CF) were administered during the operations. Hydroxycamptothecin was } \\
\text { used as peritoneal irrigation during the operations, whereas TNF, 5-FU, and CF } \\
\text { were given as intravenous injections. Ftandard of the } 7 \text { th TNM stage of } \\
\text { International Union Against Cancer (UICC) was used in our study }\end{array}$} \\
\hline
\end{tabular}

Table 2. Univariate and Multivariate Cox Proportional-hazard Regression Analysis of Receipt of IOC or POC and Cancer Survival in CRC Patients with Stage II or IIla.

\begin{tabular}{|c|c|c|c|c|}
\hline \multirow[t]{2}{*}{ Characteristic } & \multicolumn{2}{|c|}{ Univariate Analysis } & \multicolumn{2}{|c|}{ Multivariate Analysis } \\
\hline & HR (95\% CI) & P Value & HR (95\% CI) & P Value \\
\hline IOC & & $<0.001$ & & $<0.001$ \\
\hline Did not receive & 1.0 (Reference) & & 1.0 (Reference) & \\
\hline Received & $0.33(0.23-0.48)$ & & $0.30(0.19-0.48)$ & \\
\hline POC & & 0.006 & & $<0.001$ \\
\hline Did not receive & 1.0 (Reference) & & 1.0 (Reference) & \\
\hline Received & $0.62(0.45-0.87)$ & & $0.44(0.30-0.64)$ & \\
\hline TNM stage & & $<0.001$ & & $<0.001$ \\
\hline I-II & 1.0 (Reference) & & 1.0 (Reference) & \\
\hline III-IV & $2.52(1.89-3.36)$ & & $2.35(1.59-3.48)$ & \\
\hline Lymph node meta & astasis & $<0.001$ & & 0.412 \\
\hline No & 1.0 (Reference) & & 1.0 (Reference) & \\
\hline Yes & $1.70(1.28-2.25)$ & & $1.17(0.80-1.72)$ & \\
\hline Age & & 0.995 & & 0.998 \\
\hline$<50$ & 1.0 (Reference) & & 1.0 (Reference) & \\
\hline$\geq 50$ & $0.99(0.56-1.79)$ & & $1.00(0.50-2.01)$ & \\
\hline Gender & & 0.368 & & 0.769 \\
\hline Female & 1.0 (Reference) & & 1.0 (Reference) & \\
\hline Male & $0.88(0.67-1.16)$ & & $1.05(0.74-1.50)$ & \\
\hline Tumor location & & 0.694 & & 0.991 \\
\hline Colon & 1.0 (Reference) & & 1.0 (Reference) & \\
\hline Rectum & $0.94(0.70-1.27)$ & & $1.00(0.70-1.44)$ & \\
\hline Tumor size & & 0.350 & & 0.894 \\
\hline$<5 \mathrm{~cm}$ & 1.0 (Reference) & & 1.0 (Reference) & \\
\hline$\geq 5 \mathrm{~cm}$ & $1.15(0.86-1.52)$ & & $0.98(0.69-1.38)$ & \\
\hline Differentiation & & 0.024 & & 0.018 \\
\hline Good & 1.0 (Reference) & & 1.0 (Reference) & \\
\hline Moderate or Poor & $1.92(1.09-3.36)$ & & $2.16(1.14-4.06)$ & \\
\hline
\end{tabular}

\section{The association of IOC and POC with therapeutic outcomes for CRC patients}

To further analyze the CRC patient response to IOC, Kaplan-Meier analyses were performed. IOC regimens, which consisted of hydroxycamptothecin, TNF, 5-FU, and CF were administered during the operations. Hydroxycamptothecin was used as peritoneal irrigation during the operations, while TNF, 5-FU, and CF were given as intravenous injections. The results demonstrated that patients treated with IOC survived longer than the patients who were not treated with IOC during the surgery $(\mathrm{P}<0.0001$, Kaplan-Meier log rank) (Figure 1).

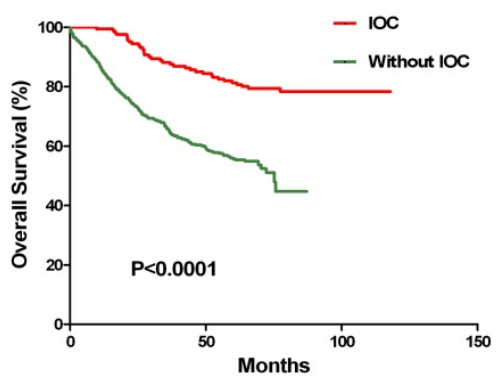

Figure 1. Association of IOC with chemotherapy outcomes for CRC patients. In the CRC patients, IOC was associated with longer survival, in the group that received chemotherapy, than was without IOC $(P<0.0001$, Kaplan-Meier log rank). 

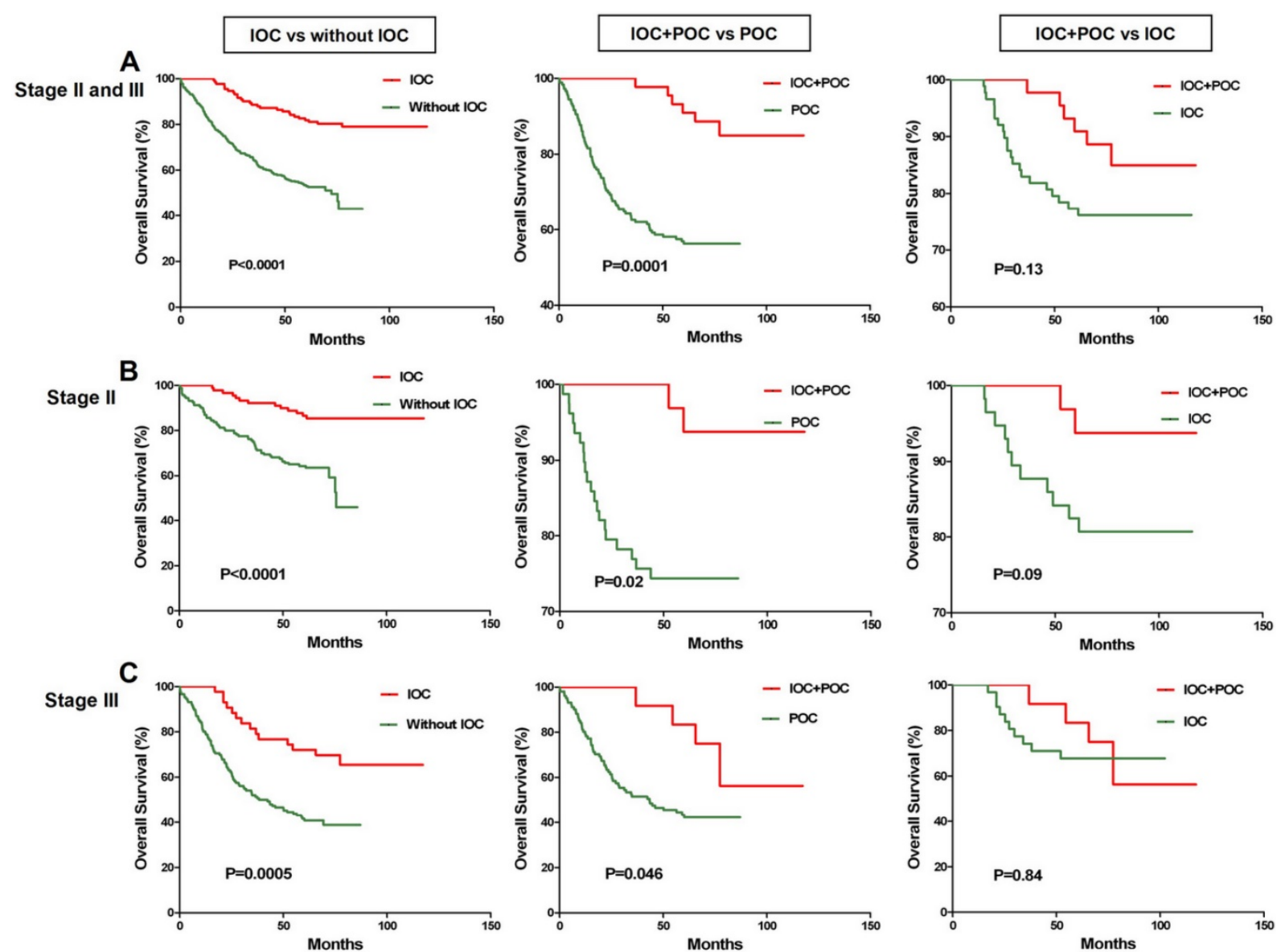

Figure 2. Association of IOC and POC with chemotherapy outcomes for the CRC patients with TNM stage II or III. A. For the 459 CRC stage II or III patients, IOC $(n=153)$ was associated with a favorable prognosis $(P<0.0001$, Kaplan-Meier log rank); CRC patients who received both IOC and POC ( $=52)$ survived longer than did patients who received only POC ( $n=195)(P=0.0001$, Kaplan-Meier log rank). B. For the $267 \mathrm{CRC}$ stage II patients, IOC ( $\mathrm{n}=107)$ was associated with a favorable prognosis $(P<0.0001$, Kaplan-Meier log rank); CRC patients who received both IOC and $P O C(n=32)$ survived longer than the patients who only received $P O C$ ( $=110)$ ( $(P=0.02$,

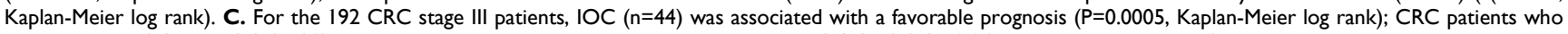
received both IOC and POC $(n=20)$ survived longer than the patients who received only POC $(n=85)(P=0.046$, Kaplan-Meier log rank).

Because the patients who received IOC might also receive POC after surgery, POC could affect the survival of these patients. This result could be caused by the IOC, POC, or a combination of both. An analysis of the response to postoperative adjuvant chemotherapy included only the TNM stage II and III patients because TNM stage I patients have excellent survival prognoses regardless of therapy, and treatment in stage IV is palliative. We analyzed the association of therapeutic outcomes in stage II and stage III CRC patients treated with postoperative adjuvant chemotherapy in the current study. The POC regimens were primarily fluorouracil based with or without leucovorin, levamisole, or cisplatin. The Kaplan-Meier analyses demonstrated that IOC was also associated with a favorable prognosis in stage II and stage III patients $(\mathrm{P}<0.0001$, Kaplan-Meier log rank), stage II patients alone $(\mathrm{P}<0.0001$, Kaplan-Meier log rank), and stage III patients alone ( $\mathrm{P}=0.0005$, Kaplan-Meier log rank) (Figure 2). The patients who received both IOC and POC survived longer than the patients who only received POC for stage II and stage III patients ( $\mathrm{P}=0.0001$, Kaplan-Meier log rank), stage II patients alone ( $\mathrm{P}=0.02$, Kaplan-Meier log rank), and stage III patients alone $(\mathrm{P}=0.046$, Kaplan-Meier $\log$ rank), but not the patients who only received IOC for stage II and stage III patients ( $\mathrm{P}=0.13$, Kaplan-Meier $\log$ rank), stage II patients alone $(\mathrm{P}=0.09$, Kaplan-Meier log rank), and stage III patients alone ( $\mathrm{P}=0.84$, Kaplan-Meier log rank) (Figure 2). Therefore, combining IOC and POC could significantly prolong the survival outcomes for CRC patients.

\section{Side effects}

The side effects of IOC involves in perforation, bleeding, and infection, while no side effects occurred in our study. Of the 193 patients who suffered from the IOC, no IOC related complications were found.

\section{Discussion}

Advances in diagnosis and surgical therapy have improved the prognosis of early CRC; however, the 
incidence of recurrence for Dukes' B and C CRC is approximately $50 \%$ [13]. The incidence of locoregional recurrence can been reduced significantly by total mesorectal excision[14]. The use of a similar approach, extensive lymph node resection, has been questioned on colon cancer[15]; surgery alone, even when macroscopically complete, is not adequate. In fact, microscopic residual disease is the cause of local recurrence in almost all patients[3]. To date, numerous adjuvant treatments have been used to prevent disease recurrence and improve survival[16-17]. Systemic chemotherapy has been shown to improve survival in stage III colon cancer[18]. Locoregional recurrence in locally advanced CRC is the result either of a tumor involving the serosa and perforation of the bowel wall or of the iatrogenic dissemination of cancer emboli that triggers locoregional tumors within 2-3 years[17]. If chemotherapy is administered with peritoneal irrigation and intravenous injection during an operation, microscopic residual tumors resulting from surgical manipulations in locally advanced colorectal cancer surgery may be eradicated.

In the present study, the clinical outcome was encouraging, and we observed no postoperative deaths. The postoperative morbidity rate was acceptable and comparable to that reported by other investigators [3, 19-20]. The surgery-related complication rate can be considered similar to that observed in major surgery studies, which supports the hypothesis that IOC does not increase the risk of postoperative complications. The mild locoregional toxicity, together with the low rate of systemic adverse effects, strengthens our opinion that IOC is a safe procedure.

Zhou et al reported a study of short-term effect analysis of intraoperative intraperitoneal perfusion chemotherapy with lobaplatin for colorectal cancer, indicating no effect on short-term recovery in patients with CRC [21]. However, survival times was not evaluated. Safety of intraoperative chemotherapy with 5-FU for colorectal cancer patients was also performed [22], and showed that it can be safely performed during colorectal surgery. In our study, we used novel regimen of hydroxycamptothecin, tumor necrosis factor (TNF), 5-fluorouracil (5-FU), and calcium folinate $(\mathrm{CF})$ for IOC, and follow up study indicated a prognostic benefit.

Currently, it is known that IOC regimens consisting of hydroxycamptothecin, TNF, 5-FU, and $\mathrm{CF}$ for the treatment of CRC patients have a prognostic benefit. The current results demonstrated that CRC patients treated with IOC had longer survival times than the patients who were not treated with IOC during the surgery. In addition, we also analyzed the association of therapeutic outcomes in stage II and stage III CRC patients treated with postoperative adjuvant chemotherapy regimens that were primarily fluorouracil based with or without leucovorin, levamisole, or cisplatin. Kaplan-Meier analyses demonstrated that IOC was also associated with a favorable prognosis in stage II and stage III patients, stage II patients alone, and stage III patients alone. The patients who received both IOC and POC survived longer than the patients who only received POC for stage II and stage III ( $\mathrm{P}=0.0001$, Kaplan-Meier log rank), stage II patients alone, and stage III patients alone, but not the patients who only received IOC in stage II and stage III, stage II patients alone, and stage III patients alone. Therefore, a combination of both IOC and POC could significantly prolong survival for CRC patients. We conclude that the IOC was effective in CRC and that IOC+POC for stage II and stage III CRC patients was a predictor of long-term disease-free survival.

\section{Conclusions}

In conclusion, the therapeutic effects of colorectal cancer by intraoperative chemotherapy with a novel regimen were enhanced, which improved the prognosis of the treated CRC patients. There is an urgent need for us to find a safer and more effective way to improve prognosis in further study of the IOC.

\section{Abbreviations}

IOC, intraoperative chemotherapy; CRC, colorectal cancer; TNF, tumor necrosis factor; 5-FU, 5-fluorouracil; $\mathrm{CF}$, and calcium folinate; POC, postoperative adjuvant chemotherapy.

\section{Acknowledgements}

We would like to express our deep and sincere gratitude to the participating patients and clinicians for their contributions to this study.

\section{Funding}

This work was financially supported by National Natural Science Foundation of China (No. 81670480, 81673206, 81570189), High level talents in Guangdong Province special support plan - Youth Science and technology innovation talents (2015TQ01R334), Major Scientific Research Projects at Provincial Level in Guangdong General University (2017KZDXM068), Innovation Team Project of Guangzhou Education Bureau (201831828), and Project of Guangzhou Chinese Medicine Bureau (20162095). The authors have declared that no conflict of interest exists. 


\section{Authors' contributions}

Zhihua Liu, Yifeng Zou and Xinke Zhou conceived and designed the study. Yinghai Tian, Weipeng Li, Chao Li, Hongcheng Lin, Yuming Rong, Huan Zhao, Min Liu and Jinsheng Weng analyzed the data and prepared the manuscript. Ting Liu, Xiaomei $\mathrm{Li}$, Chao Lei, and Chen Li participated in study design. Zhihua Liu, Weipeng $\mathrm{Li}$ and Xinke Zhou provided valuable discussions with regard to clinical correlates. All authors read and approved the final manuscript.

\section{Ethics approval and consent to participate}

Informed consent has been obtained from all patients and all procedures were approved by the related Institute Ethics Committee.

\section{Competing Interests}

The authors have declared that no competing interest exists.

\section{References}

1. Jemal A, Siegel R, Ward E, Hao Y, Xu J, Murray T, et al. Cancer statistics, 2008. CA Cancer J Clin. 2008; 58: 71-96.

2. Sung JJ, Lau JY, Goh KL, Leung WK. Increasing incidence of colorectal cancer in Asia: implications for screening. Lancet Oncol. 2005; 6: 871-6.

3. Pilati P, Mocellin S, Rossi CR, Foletto M, Campana L, Nitti D, et al. Cytoreductive surgery combined with hyperthermic intraperitoneal intraoperative chemotherapy for peritoneal carcinomatosis arising from colon adenocarcinoma. Ann Surg Oncol. 2003; 10: 508-13.

4. de Gramont A, Van Cutsem E, Schmoll HJ, Tabernero J, Clarke S, Moore MJ, et al. Bevacizumab plus oxaliplatin-based chemotherapy as adjuvant treatment for colon cancer (AVANT): a phase 3 randomised controlled trial. Lancet Oncol. 2012; 13: 1225-33.

5. Sanoff HK, Carpenter WR, Sturmer T, Goldberg RM, Martin CF, Fine JP, et al. Effect of adjuvant chemotherapy on survival of patients with stage III colon cancer diagnosed after age 75 years. J Clin Oncol. 2012; 30: 2624-34.

6. Dewdney A, Cunningham D, Tabernero J, Capdevila J, Glimelius B, Cervantes A, et al. Multicenter randomized phase II clinical trial comparing neoadjuvant oxaliplatin, capecitabine, and preoperative radiotherapy with or without cetuximab followed by total mesorectal excision in patients with high-risk rectal cancer (EXPERT-C). J Clin Oncol. 2012; 30: 1620-7.

7. Yothers G, O'Connell MJ, Allegra CJ, Kuebler JP, Colangelo LH, Petrelli NJ, et al. Oxaliplatin as adjuvant therapy for colon cancer: updated results of NSABP C-07 trial, including survival and subset analyses. J Clin Oncol. 2011; 29: 3768-74

8. Begos DG, Ballantyne GH. Regional chemotherapy for colorectal liver metastases: thirty years without patient benefit. J Surg Oncol. 1994; 56: 139-44.

9. Lorenz M, Muller HH, Schramm H, Gassel HJ, Rau HG, Ridwelski K, et al Randomized trial of surgery versus surgery followed by adjuvant hepatic arterial infusion with 5-fluorouracil and folinic acid for liver metastases of colorectal cancer. German Cooperative on Liver Metastases (Arbeitsgruppe Lebermetastasen). Ann Surg. 1998; 228: 756-62.

10. Patt YZ. Regional hepatic arterial chemotherapy for colorectal cancer metastatic to the liver: the controversy continues. J Clin Oncol. 1993; 11: 815-9.

11. Kemeny N, Daly J, Oderman P, Shike M, Chun H, Petroni G, et al. Hepatic artery pump infusion: toxicity and results in patients with metastatic colorectal carcinoma. J Clin Oncol. 1984; 2: 595-600.

12. Rashidi $B$, An Z, Sun FX, Moossa AR, Hoffman RM. Antimetastatic intraoperative chemotherapy of human colon tumors in the livers of nude mice. Clin Cancer Res. 2000; 6: 2464-8.

13. Galandiuk S, Wieand HS, Moertel CG, Cha SS, Fitzgibbons RJ, Jr., Pemberton $\mathrm{JH}$, et al. Patterns of recurrence after curative resection of carcinoma of the colon and rectum. Surg Gynecol Obstet. 1992; 174: 27-32.

14. MacFarlane JK, Ryall RD, Heald RJ. Mesorectal excision for rectal cancer. Lancet. 1993; 341: 457-60.

15. Tentes AA, Mirelis C, Karanikiotis C, Korakianitis O. Radical lymph node resection of the retroperitoneal area for left-sided colon cancer. Langenbecks Arch Surg. 2007; 392: 155-60.

16. August DA, Ottow RT, Sugarbaker PH. Clinical perspective of human colorectal cancer metastasis. Cancer Metastasis Rev. 1984; 3: 303-24.
17. Tentes AA, Spiliotis ID, Korakianitis OS, Vaxevanidou A, Kyziridis D. Adjuvant perioperative intraperitoneal chemotherapy in locally advanced colorectal carcinoma: preliminary results. ISRN Surg. 2011; 2011: 529876.

18. Laurie JA, Moertel CG, Fleming TR, Wieand HS, Leigh JE, Rubin J, et al. Surgical adjuvant therapy of large-bowel carcinoma: an evaluation of levamisole and the combination of levamisole and fluorouracil. The North Central Cancer Treatment Group and the Mayo Clinic. J Clin Oncol. 1989; 7: $1447-56$

19. Witkamp AJ, de Bree E, Kaag MM, Boot H, Beijnen JH, van Slooten GW, et al. Extensive cytoreductive surgery followed by intra-operative hyperthermic intraperitoneal chemotherapy with mitomycin-C in patients with peritoneal carcinomatosis of colorectal origin. Eur J Cancer. 2001; 37: 979-84.

20. Witkamp AJ, de Bree E, Kaag MM, van Slooten GW, van Coevorden F, Zoetmulder FA. Extensive surgical cytoreduction and intraoperative hyperthermic intraperitoneal chemotherapy in patients with pseudomyxoma peritonei. Br J Surg. 2001; 88: 458-63.

21. Zhou HT, Jiang J, Guan X, Su H, Liang JW, Pei W, et al. The short-term effect analysis of intraoperative intraperitoneal perfusion chemotherapy with lobaplatin for colorectal cancer. J BUON. 2019; 24: 442-8.

22. Zhang RX, Lin JZ, Lei J, Chen G, Li LR, Lu ZH, et al. Safety of intraoperative chemotherapy with 5-FU for colorectal cancer patients receiving curative resection: a randomized, multicenter, prospective, phase III IOCCRC trial (IOCCRC). J Cancer Res Clin Oncol. 2017; 143: 2581-93. 